OPEN ACCESS

Edited by: Han-Yu Shih, National Eye Institute (NEI), United States

Reviewed by: Ana Maria Gamero, Temple University, United States Daniella Schwartz, National Institute of Allergy and Infectious Diseases $(\mathrm{NIH})$, United States

*Correspondence: Wenda Gao wendagao01@gmail.com

${ }^{+}$These authors have contributed equally to this work

Specialty section:

This article was submitted to Cytokines and Soluble Mediators in Immunity, a section of the journal

Frontiers in Immunology

Received: 16 August 2021 Accepted: 06 December 2021 Published: 21 December 2021

Citation: Yan J, Yu J, Liu K, Liu Y, Mao C and Gao W (2021) The Pathogenic Roles of

IL-22 in Colitis: Its Transcription Regulation by Musculin in T Helper Subsets and Innate Lymphoid Cells.

Front. Immunol. 12:758730. doi: 10.3389/fimmu.2021.758730

\section{The Pathogenic Roles of IL-22 in Colitis: Its Transcription Regulation by Musculin in T Helper Subsets and Innate Lymphoid Cells}

\author{
Jun Yan ${ }^{1 \dagger}$, Jing Yu ${ }^{1 \dagger}, \mathrm{Ke} \mathrm{Liu}^{1,2}$, Yijia Liu ${ }^{1,2}$, Changchuin $\mathrm{Mao}^{3}$ and Wenda Gao ${ }^{3 *}$ \\ 1 State Key Laboratory of Trauma, Burns and Combined Injury, Department of Special War Wound, Research Institute of \\ Surgery, Daping Hospital, Army Medical University, Chongqing, China, 2 School of Life Science and Engineering, Southwest \\ Jiaotong University, Chengdu, China, ${ }^{3}$ Antagen Pharmaceuticals, Boston, MA, United States
}

IL-22 plays a crucial role in promoting inflammation, antimicrobial immunity and tissue repair at barrier surfaces. The role of IL-22 in colitis is still controversial: while IL-22 has a protective effect on gut epithelium in acute injuries, it also enhances colitis in a contextdependent manner. Here, we summarize the Yin and Yang of IL-22 in colitis. Particularly, we emphasize the role of innate lymphoid cells (ILCs) in IL-22 production and regulation. A previously underappreciated transcription factor, Musculin (MSC), has been recently identified to be expressed in not only Th17 cells, but also ROR $\mathrm{t}^{+} / \mathrm{ld} 2^{+} \mathrm{IL}-22$-producing group 3 ILCs in the gut of naive mice. We hypothesize that the co-expression and interaction of MSC with the key transcription repressor ld2 in developing lymphoid cells (e.g., in LTi cells) and ILC precursors might fine tune the developmental programs or regulate the plasticity of adaptive Th subset and innate ILCs. The much-elevated expression of IL-22 in MSC-/- ILC3s suggests that MSC may function as: 1) a transcription suppressor for cytokines, particularly for IL-22, and/or 2) a gatekeeper for specific lineages of Th cells and innate ILCs as well. Amelioration of colitis symptoms in MSC-/- mice by IL-22-blocking agent IL-22BP-Fc suggests a counterintuitive pathogenic role of IL-22 in the absence of MSC as a checkpoint. The theory that exuberant production of IL-22 under pathological conditions (e.g., in human inflammatory bowel disease, IBD) may cause epithelial inflammation due to endoplasmic reticulum (ER) stress response is worth further investigation. Rheostatic regulation of IL-22 may be of therapeutic value to restore homeostatic balance and promote intestinal health in human colitis.

Keywords: IL-22, Musculin, ILC3, colitis, mucosal immunity, Th17, transcription factor, ROR $\gamma \mathrm{t}$

\section{INTRODUCTION}

Since its discovery more than two decades ago (1), Interleukin-22 (IL-22) has been extensively studied for its roles in maintaining mucosal barrier integrity, antimicrobial defense, cellular proliferation and inflammation. The beneficial and pathogenic roles of IL-22 in various disease settings, and in intestine homeostasis in particular, have been very well summarized in quite a few 
excellent reviews (2-4). Of note, knowledge on IL-22 cellular sources, receptor signaling, cytokine induction and feedback regulation of its production, as well as IL-22 stimulatory functions on antimicrobial molecules has greatly promoted our understanding of this crucial cytokine in intestinal epithelium regeneration and barrier protection, as well as pathobiology in human diseases, such as rheumatoid arthritis, psoriasis, interstitial lung diseases and colon cancer (5).

On the other hand, the differentiation, plasticity and phenotype maintenance of innate lymphoid cells (ILCs) in IL-22 production is still an uncharted area, awaiting more detailed analysis on the transcription factors (TF) that help define their developmental pathways and phenotypic stability. We propose that pharmacological interventions on specific regulating TFs in ILCs, rather than targeting the secretary products of ILCs, which usually have multifaceted functions (6), could lead to more stable beneficial outcomes in human diseases, maintained by long-lasting ILC populations. For that purpose, herein we first summarize the current knowledge on major TFs involved in the differentiation of both $\mathrm{T}$ helper cell subsets and their innate lymphoid counterparts. Then, we will introduce a previously underappreciated transcription repressor, Musculin (MSC), with accumulating evidence for its critical roles in IL-22 regulation, particularly in ILCs. Discoveries in MSC-/- mice support the emerging theory that IL-22 overproduction in colitis underlies intestinal epithelial cell stress response, and call for cautious re-evaluation on IL-22 supplementation therapy for human IBD.

\section{T HELPER CELL DIFFERENTIATION AND SHARED TFs IN ILCs}

Cellular differentiation requires the precise actions of lineagespecifying TFs. In the immune system, naïve CD4+ T helper cells differentiate into distinct lineages with unique cytokine profiles. Besides T helper type 1 (Th1), Th2, Th17 and T regulatory (Treg) cells, with their master TFs being T-bet, GATA-3, ROR $\gamma$ t/ROR $\alpha$ and Foxp3, respectively, newer subsets such as Th9, T follicularhelper (Tfh), as well as Th22 cells have also been recognized (7-13). Although much has been known about the extracellular cytokine cues on the differentiation of classical $\mathrm{T}$ helper subsets (Th1, Th2, Th17 and Treg) $(7-9,14)$, it remains to be pinpointed, besides those master TFs, what Th-specific intracellular regulators contribute to maintaining the phenotype and function of Th subsets.

While $\mathrm{T}$ helper cells are an established realm of adaptive immunity, innate lymphoid cells (ILCs) have recently come into focus $(15,16)$. ILCs lack antigen specificity, but are particularly enriched at barrier and mucosal surfaces to regulate tissue homeostasis and immune responses. ILC family consists of a variety of developmentally-related innate immune cells, as they all depend on the transcriptional repressor, inhibitor of DNA binding 2 ( $\operatorname{Id} 2)$, as well as on the common cytokine receptor $\gamma$-chain for their development (17, 18). Based on their cytokine secretion profiles, ILCs fall into three major groups (15): ILC1s, ILC2s and ILC3s, in parallel with the prominent CD4+ Th1, Th2, and Th17 subsets, and express similar transcription factors and cytokines $(19,20)$. Namely, ILC1s express T-bet and produce interferon- $\gamma($ IFN- $\gamma$ ) (21); ILC2s are enriched with higher levels of GATA-3 and express IL-5, IL-9 and IL-13 (22, 23); ILC3s are featured with the transcription factor ROR $\gamma$ t and secrete the Th17 cytokines IL-17 and IL-22 (24). A possible fourth group IL-10-secreting regulatory ILCs (ILCregs) with unique surface markers and transcription signatures has been identified in the intestines of mice and humans (25). ILCregs inhibit ILC1- and ILC3induced intestinal inflammation in an IL-10-dependent manner, yet unlike Tregs, they are highly enriched in Id 3 but do not express Foxp3 (25). The existence of ILCregs is still controversial (26), as other groups suggested that they may originate from IL-10-secreting ILC2s $(27,28)$.

ROR $\gamma t+$ group 3 ILCs (ILC3s) are the most heterogeneous ILC population and can be further divided into several subsets: CD4+CD3- LTi (lymphoid tissue inducer) cells that are primarily involved with secondary lymphoid organ formation during embryogenesis, and upon stimulation are able to secrete IL-17A and IL-22 (24). A second subset typically found in mucosal tissues produces IL-22 but not IL-17A, also known as NK22 cells and ILC22s (29). The third subset of ILC3s secretes IFN- $\gamma$, IL-17A, and IL-22, and was found to play a pathogenic role in murine innate colitis (30). Thus, the main cytokine produced by ILC3s is IL-22 and some populations of ILC3s also produce IL-17. Akin to their Th counterparts, considerable plasticity exists among ILC groups (16), and trans-differentiation of ILCs could underlie certain diseases (31). The major TFs involved in $\mathrm{Th}$ and ILC differentiation are summarized in Figure 1.

\section{NETWORKS OF TFS IN LINEAGE SPECIFICATION AND PLASTICITY OF TH CELLS AND ILCs}

As mentioned earlier, "master TFs" were thought to be the main determining factors for specific cell lineages. It is nevertheless becoming more and more appreciated that networks of regulatory TFs collectively contribute to the differentiation program and lineage maintenance. To reveal any additional players other than the "master TFs", high-throughput microarray and expression profiling technologies along with functional knockout and knockin strategies need to be exploited to delineate the complex regulatory networks of TFs co-expressed within a differentiating or trans-differentiating cell $(32,33)$.

A good example for such lineage plasticity is between Treg and Th17 cells. While TGF- $\beta$ induces naïve $\mathrm{T}$ cells to differentiate into Tregs, in the presence of TGF- $\beta$ and IL- 6 or IL21 Treg differentiation is suppressed whereas Th17 development is promoted $(14,34)$. The reciprocal developmental pathways for Treg vs. Th17 suggest that these two important Th subsets are interrelated and can transform to each other under specific conditions. Indeed, Treg trans-differentiation has been widely 

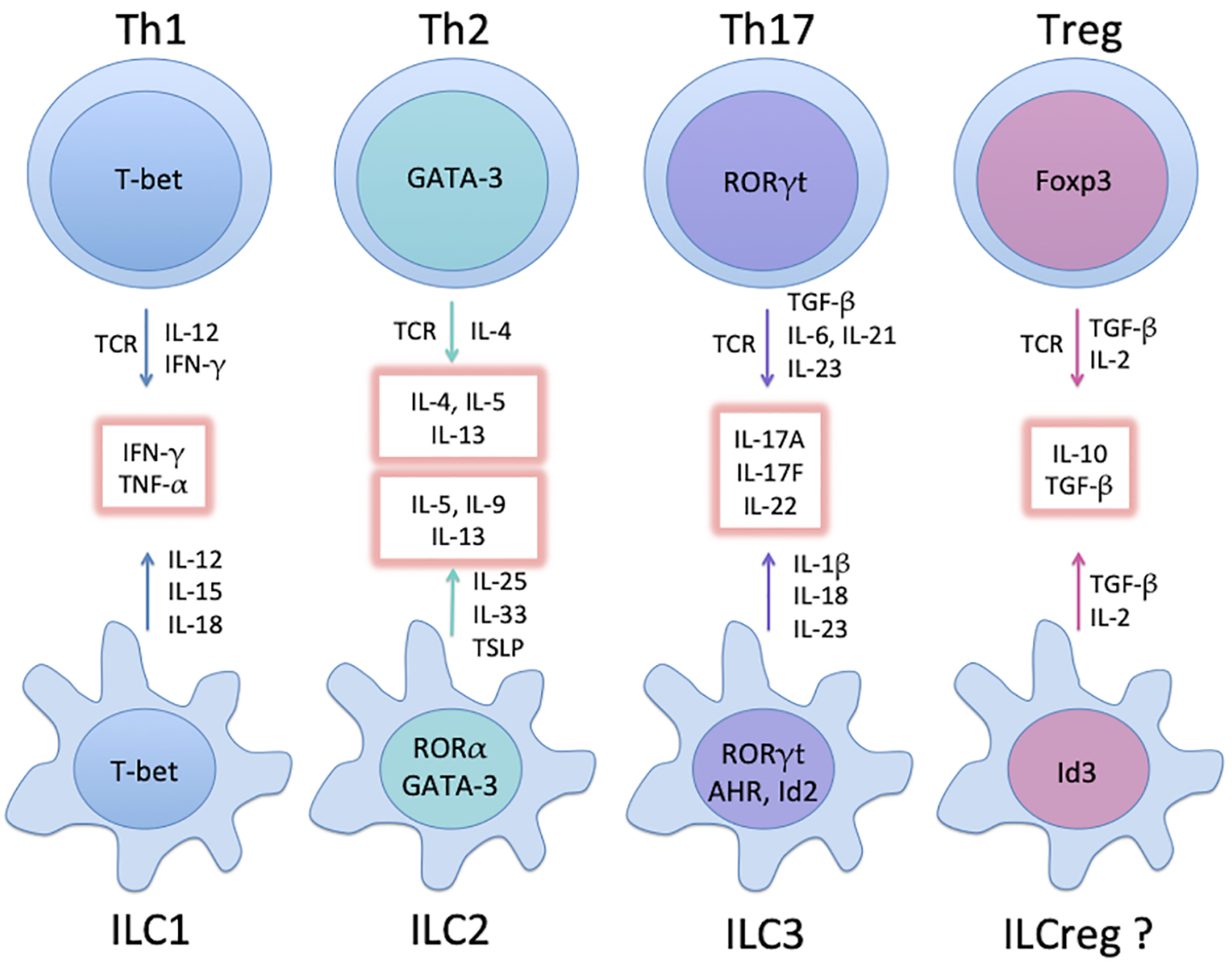

FIGURE 1 | Major groups of Th subsets and ILCs, and their master TFs. Groups of mature ILCs mirror Th subsets with similar cytokine profiles (glowing boxes). Not depicted here are common helper-ILC progenitors (CHILPS) relying on Id2 and GATA-3 for differentiation. While ILC1 and ILC3 develop directly from the CHILPS upon expression of T-bet and RORyt, respectively, further increase in GATA-3 expression leads to the development of the ILC2 precursor (ILC2P) and subsequently ILC2. Note that the existence of ILCreg is still controversial (see text).

observed in autoimmune diseases, such as juvenile arthritis, type 1 diabetes and multiple sclerosis (35), as well as in transplant rejection (36). In an inflammatory microenvironment enriched with IL-6 and IL-23 cytokines, "ex-Tregs" are converted into IL$17+$ or IFN- $\gamma+$ effectors and are functionally indistinguishable from Th17 or Th1 cells, respectively (37).

Likewise, Th17 cells also demonstrate considerable plasticity and readily downregulate IL-17 secretion and turn into Th1-like cells in autoimmune and chronic inflammatory conditions (38-40). In the tumor microenvironment (TME), tumor-induced Foxp3negative IL-17A+ Th17 cells decline in number at later time points, concomitant with the increase of "ex-Th17" Foxp3+IL-17Asuppressive tumor-associated Tregs (41). Th17-to-Treg transdifferentiation is fostered by TGF- $\beta$ and tumor-derived $\mathrm{PGE}_{2}$ (41). While TGF- $\beta$ in conjunction with IL- 6 and IL-23 promotes Th17 cell differentiation and IL-17A secretion, the presence of $\mathrm{PGE}_{2}$ inhibits this effect and promotes the conversion of Th17 cells into Foxp3+IL-17A- cells (41). Thus, TME-derived extracellular immunosuppressive factors play a major role in reprogramming
Th cells (41). But what intracellular factor(s) determining the fate of metastable Foxp3+IL-17A+ cells in the late stage at the fork of transdifferentiation is largely unknown.

Like Th cells, plasticity of ILC subsets in reflection of the tissue microenvironment has been a hot area of intensive studies (16). Uncovering additional TFs driving such transdifferentiation in both Th subsets and ILCs is of great importance in understanding the phenotype stability of these immune cells. Modulating TFs that are associated with this process could have significant therapeutic value for human diseases. This is because that unlike in naive animals for de novo disease induction, in human diseases, lineage specification of pathogenic Th/ILC cells may have already taken place at the time when symptoms are manifested. Thus, the window of opportunity for pharmacological intervention may exist at the stage of lineage maintenance and trans-differentiation. In light of this, we now introduce a novel TF, Musculin, which could be implicated in regulating Th and ILC stability, particularly in connection with IL-22 production in ILC3s. 


\section{MUSCULIN (MSC) AS AN EMERGING TF FINE-TUNING BOTH TH CELLS AND ILCs}

\section{MSC in Non-Lymphoid Cells}

MSC (also known as MyoR (42), ABF-1 and bHLHa22) is a basic helix-loop-helix transcription repressor that is involved in the regulation of muscle development $(42,43)$. MSC can bind to an E-box motif (CANNTG) either as a homodimer or as a heterodimer with E2A gene products, where the dimers bind the same E-box sequence as myogenic bHLH/E protein heterodimers, but MSC functions as a potent transcriptional repressor that inhibits myogenesis and E-box-dependent muscle gene activation (44). MSC shows two isoforms (45): a 201 aa protein (MSC 1a) and an equally effective 180 aa short form (MSC 1b), with a repressor domain between aa 158-173. Although early studies indicate that MSC has a muscle-specific, developmental stage-restricted expression profile, more recent studies support the notion that "Musculin" could very well be a misnomer, as its expression is also found in several adult tissues of non-muscle lineage. For instance, MSC is expressed in kidney side population (SP) cells, which are enriched with stem cells, and can regulate their function (46).

\section{MSC in Lymphocyte Differentiation}

In lymphoid compartment, human MSC is known as activated B cell factor-1 (ABF-1). MSC facilitates the formation of germinal center B cells and memory B cells, but inhibits the differentiation of antibody-secreting plasma cells (47). In Hodgkin's lymphoma, $B$ cell-specific transcription program is interrupted by overexpression of ABF-1 (a.k.a. MSC) and Id2, which antagonize the function of the transcription factor E2A in $\mathrm{B}$ cell lineage determination. As a result, expression of $\mathrm{B}$ cell specific genes is lost, whereas genes normally not associated with the B cell lineage are upregulated (48).

The expression and regulatory roles of MSC in T cells, especially CD4+ T helper cells, await further clarifications. One group reported that MSC is induced in murine Tfh but not Th17 subset, yet MSC is not required for the differentiation of Tfh cells, nor plays a significant role in antibody production in response to T-dependent antigens (49). This is in contradiction to the finding that MSC is selectively expressed in human Th17, but not Tfh cells, and inhibits Th17 proliferative response to IL-2 (50). Furthermore, MSC is found to be preferentially upregulated in peripherally-induced Tregs (iTregs) than in other Th subsets, and it promotes unidirectional development of iTregs by inhibiting the Th2 differentiation pathway (51). It is likely that confusion in the expression profiles of MSC stems from the various conditions of in vitro Th cell differentiation.

To avoid the potentially non-physiological anti-CD3/CD28stimulated differentiation, in our own work, we generated alloantigen-activated Th subsets whose TCR recognizes $\alpha$ chain of I-E in the context of $I-A^{b}$. MSC is dramatically upregulated in alloantigen-stimulated murine Th17, marginally in Th1, but not in Th0, Th2, or iTreg subsets (52). APC-derived IL-23 has a salutary role in MSC induction. In the in vitro activation system with anti-CD3/CD28 in the absence of antigen-presenting cells (APC), Th17 cells also express more MSC than Th0 and Th1 cells, but only at later time points (6-8 days) (52). Such kinetics suggests that most researchers could have missed MSC in fully developed Th17 cells if they performed Th subset differentiation in the absence of APC in the first 2-4 days $(49,51)$. In addition, we found that when naïve CD4 +CD62 $\mathrm{L}^{\text {hi }} \mathrm{GFP}$ - cells from IL-17A ${ }^{\text {GFP }}$ knockin mice were differentiated and FACS-sorted based on GFP intensities, MSC correlates with stronger Th17 phenotypes (higher IL-17A/IL17F/ROR $\gamma t / R O R \alpha / I d 2)$ (52). Clearly, MSC is a Th17-specific TF in both human (50) and mouse (52) systems.

\section{MSC in Th Lineage Commitment and Stability: Effects on the Promoters of Master TFs RORC Promoter}

A glimpse of transcription regulation on the promoter activities of master TFs may give clues on how MSC may affect Th lineage commitment and phenotype maintenance. For instance, transcription of ROR $\gamma$ t in the developing Th17 cells is regulated by E-proteins, and E-box binding sites are essential for ROR $\gamma$ t promoter activities (53). TGF- $\beta$ and IL-6, the cytokines promoting Th17 differentiation (14), sequentially induce the expression of E-proteins and Id3 (53). Id proteins are a family of transcription regulators that bind to E-proteins, interfering with their DNA-binding capacity and transcriptional activities (54-57). Within this family, Id 2 and Id 3 are the main players in the regulation of E-protein function during lymphoid development $(58,59)$. Both of these Id proteins, as well as MSC, are expressed in Th17 cells $(52,53)$. As MSC interacts with Id 2 to antagonize the function of E2A $(44,48)$, it is highly possible that MSC could directly or indirectly participate in Th17 lineage specification through modulating E2A activities on ROR $\gamma \mathrm{t}$ transcription. Particularly, as MSC is expressed at a later time point during Th17 differentiation (52), MSC is more likely involved in Th17 stability. Future studies using promoterluciferase reporter assay and comparing MSC+/+ and MSC-/Th17 cells may reveal the role of MSC in regulating ROR $\gamma t$ transcription and Th17 differentiation and stability.

\section{FOXP3 Promoter}

Similar in Th17 cells, differentiation of Tregs is also controlled by E2A and Id3 (60). Enhanced binding of E2A to the FOXP3 promoter increases Foxp 3 transcription, and Id 3 potentiates this process by mitigating the inhibitory effect of GATA-3 at the FOXP3 promoter (60). Inflammation-induced Treg-specific expression of Id 2 reduces Foxp3 expression by blocking E2A binding to its promoter, leading to the induction of Th17-related cytokines and the "ex-Treg Th17" phenotype (61). Thus, it is suggested that Id 2 triggered by inflammatory stimuli destabilizes Tregs into Th17 cells (61).

Although we were unable to detect MSC expression in natural Tregs (nTregs) or alloantigen-stimulated in vitro induced Tregs (iTregs) (52), nTregs stimulated by anti-CD3/CD28 microbeads in the presence of IL- 6 do express increased amounts of MSC, concomitant with the expression of IL-17A/F and ROR $\gamma \mathrm{t} / \mathrm{ROR} \alpha$, 
compared to resting nTregs or nTregs stimulated by anti-CD3/ CD28 beads only (Supplementary Figure 1). In addition, GFPlabeled nTregs from Foxp3GFP knockin mice when adoptively transferred into RAG-1-/- mice can be recovered after 2 weeks into post-RAG-GFP+ and post-RAG-GFP- populations. Compared with nTregs, the post-RAG-GFP- cells lost Foxp3 expression, but upregulated IL-17A/F, ROR $\gamma \mathrm{t} / \mathrm{ROR} \alpha$ and Id 2 expression. Most interestingly, while the post-RAG-GFP+ cells had an intermediate presentation of Foxp3 and Th17 signature genes compared with nTregs and post-RAG-GFP- cells, they expressed the highest amount of MSC (hundreds of fold increase) among the three groups of cells (Supplementary Figure 2). Thus, MSC triggered by IL-6 or homeostatic proliferation in lymphopenic hosts could de-stabilize Tregs for Th17 programming, possibly through interaction with Id proteins to modulate E2A transcription regulation of master TFs. It would be highly intriguing to test this hypothesis by transferring MSC-/- nTregs from Foxp3GFP $\times$ MSC-/- mice into the RAG-1-/- mice, and determine the fate of these nTregs during homeostatic proliferation. These experiments are currently underway.

\section{AHR Promoter}

Besides the promoters of RORC (53) and FOXP3 (60) that contain E-boxes, a conserved canonical E-box sequence is also present in the promoter of aryl hydrocarbon receptor (AhR) (62). Chromatin immunoprecipitation (ChIP) experiments showed that the E-box motif in the promoter region of $A H R$ is specifically associated with Myc and its heterodimeric partner Max (62), both of which are members of the basic helix-loophelix (bHLH) leucine zipper (LZ) family of transcription factors. Homodimerization of Max or heterodimerization between Max and Myc allows these proteins to bind the E-box (CACGTG) (63). It remains to be determined whether MSC binds the E-box sequence in the $A H R$ promoter.

Our own work using Foxp3GFP mice showed that alloantigenstimulated iTregs isolated by GFP-based FACS sorting from the primary mixed lymphocyte reaction (MLR) expressed none to low levels of MSC and AhR, but their expression levels significantly increased during the secondary MLR, concomitant with dramatic expression of Th17 effector molecule osteopontin (OPN) and greatly diminished expression of Treg-associated CCR7 (64) (Supplementary Figure 3). This suggests that co-expression of MSC and AhR underlines the metastability and transition of exTregs into Th17-like effector cells.

As E2A plays a crucial role in orchestrating lymphoid differentiation in early multipotent progenitors $(57,65)$, factors affecting E2A activities could have significant impact on the genes regulated by E2A. ChIP coupled with high-throughput genome-wide sequencing in human myoblasts revealed that MSC shows widespread binding at tens of thousands of genomic sites. Its consensus recognition motif has the palindromic sequence of "CCAGCTGG" (66), overlapping with the canonical E-box sequence "CAGCTG" (67). Thus, MSC could obstruct E2A binding to the E-box sites in the promoters of the master TFs, as a major mechanism of regulating Th differentiation and stability.
Summarizing what is known about MSC expression and function, we propose a model of MSC regulation of the differentiation and/or stability of Th subsets (Figures 2, 3, and Supplementary Figures 1-4). MSC is maximally induced in Th17 cells at later time points in the presence of APC-derived IL-23, and marginally induced in Th1 cells, but not in Th0, Th2 or iTreg cells (52). The major role of MSC in specifying Th differentiation is to suppress E-box-dependent activation of master TFs. For instance, it blocks unorthodox expression of GATA-3-mediated Th2 gene IL-4 in Th17 cells (Supplementary Figure 4). MSC also suppresses the transcriptional activities of mater TFs in their corresponding host cells, such as T-bet in Th1 and ROR $\gamma$ t in Th17 cells, so that these cells are not exhausted. MSC-/- Th1 cells secrete huge amounts of IFN- $\gamma$, but are prone to apoptosis (Supplementary Figure 4). Note that MSC is not expressed in Foxp3+ iTregs per se, and could be involved in Treg trans-differentiation into Th17 cells (Figure 3).

Because of the antagonizing effects of cytokines from opposing lymphocyte subsets, caution should be taken in interpreting the data. For instance, $\mathrm{Wu}$ et al. proposed that MSC is required for iTreg induction by repressing GATA-3mediated Th2 programming, as MSC deficiency reduced expression of the master TF Foxp3 in iTreg cells and induced Th2 development even under the conditions promoting for iTreg differentiation (51). Of note, the authors did not separate the Foxp3GFP+ cells from Foxp3GFP-cells during iTreg induction. It is likely that IL-4 boosted by MSC deficiency (51) from Foxp3GFP- cells suppresses Foxp3 expression in the nearby Foxp3GFP+ cells (68) in a paracrine mode. By a similar fashion, Id 3 was claimed to be required for Foxp3 induction, as Id3-/- T cells failed to generate iTregs in the presence of TGF$\beta 1$ (60). However, when anti-IL-4 was added to the system, induction of iTreg by Id3-/- T cells was restored to the level by Id $3+/+$ T cells (60). Perhaps more definitive answer to probe for the role of additional TFs in regulating the differentiation and stability of Th subsets and ILCs should come from the knockin system where MSC, or Id3 or other potential factors are expressed in a linked manner with the master TFs, hence cell intrinsic effect of the regulators can be studied on a per cell basis, without the interference from exogenous cytokines.

\section{Possible Role of MSC in ILC3 Differentiation and Cytokine Secretion}

Recently, AhR has been implicated as a master regulator involved in the postnatal maintenance of intestinal ROR $\gamma t+$ ILCs and intraepithelial lymphocytes (69). Various populations of IL-22producing ILCs all express ROR $\gamma \mathrm{t}$ and $\mathrm{AhR}$, and all have a shared requirement for IL-23 to produce IL-22 $(24,70)$. As all the ILC progenitors express $\mathrm{Id} 2$ (71) and MSC interacts with $\mathrm{Id} 2$ to antagonize the function of E2A $(44,48)$, MSC could directly or indirectly participate in ILC development through modulating E2A activities on ROR $\gamma$ t and AhR transcription, as discussed above. More studies are needed to reveal the role of MSC in ILCs.

In our own work, we examined $\operatorname{Rorc}(\gamma \mathrm{t})^{+/ \mathrm{GFP}}$ mice whose ROR $\gamma \mathrm{t}^{\mathrm{GFP}+}$ cells are highly enriched in early thymic CD4+ cells and small groups of LTi/Th17/ILCs found in the gut-associated lymphoid tissues (GALT) (72). In Peyer's patches (PP), memory 


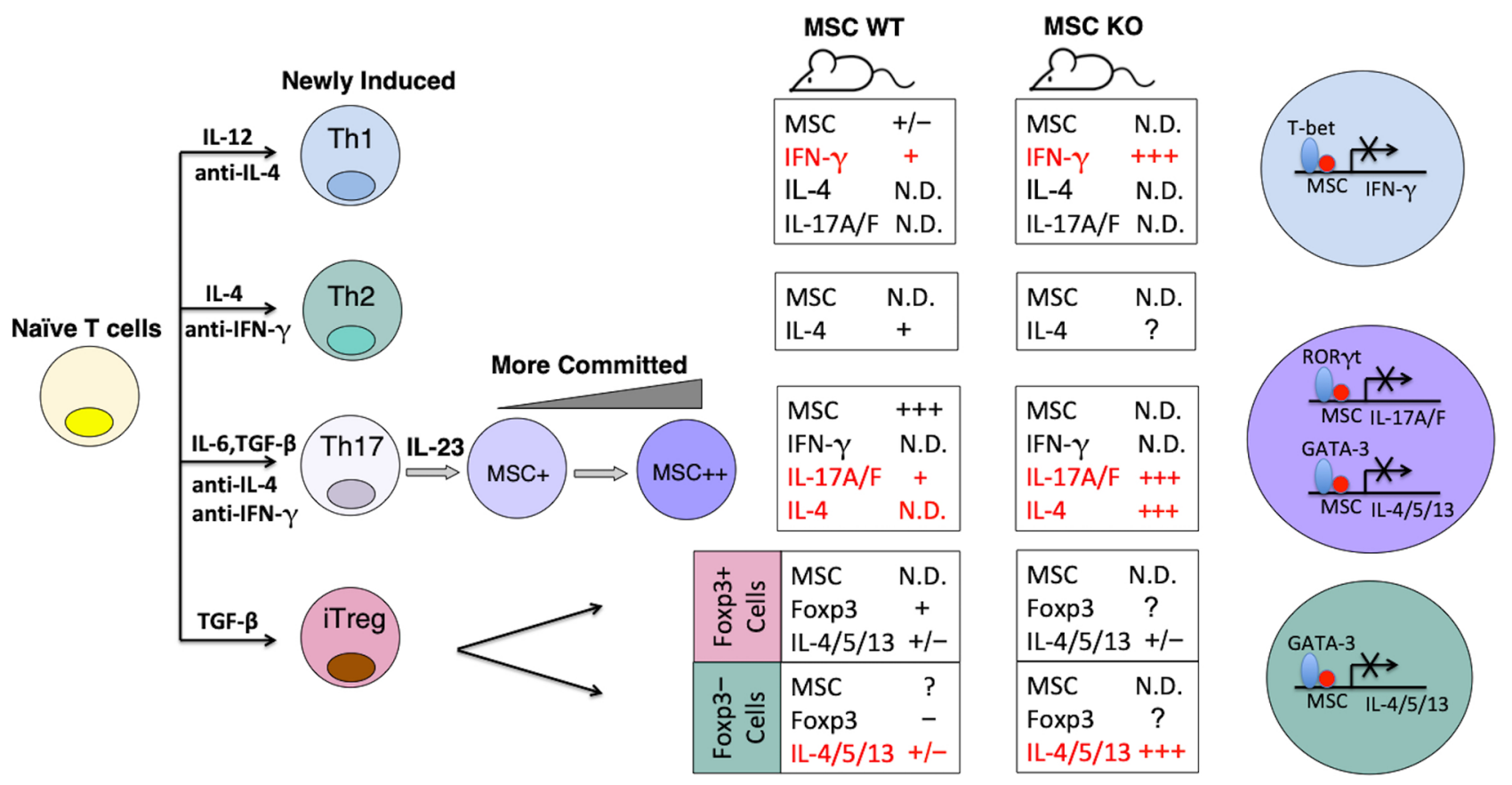

FIGURE 2 | Model of MSC regulation of the differentiation and stability of Th subsets. The expression levels of MSC correlate with stronger Th17 phenotypes. Summaries of gene expression patterns in the boxes correspond to the Th subsets on the left. N.D, not detectable. The major role of MSC in specifying Th differentiation is to suppress E-box-dependent activation of master TFs.

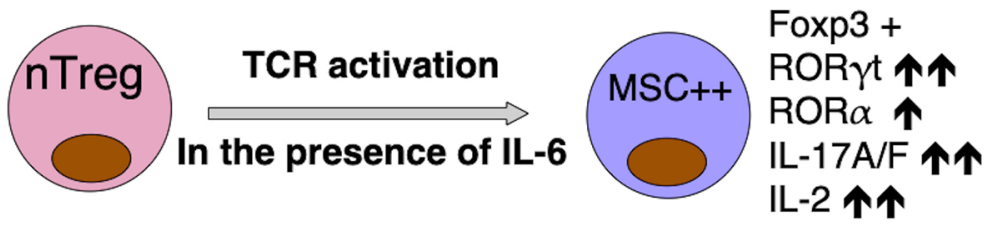

Foxp3 +++ MSC RORyt - nTreg ROR $\alpha$ IL-17A/F IL-2 -
Homeostatic

Proliferation

2 weeks
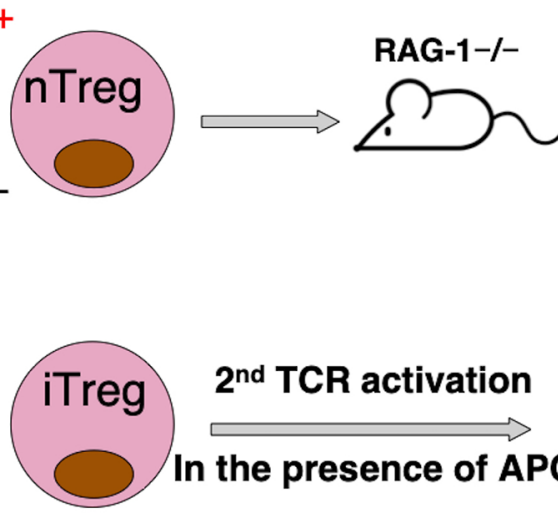

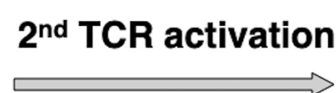

In the presence of APC

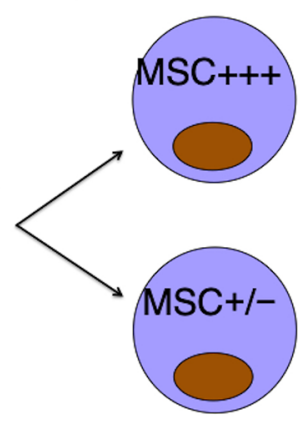

Foxp3 +

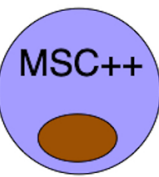

AHR $\uparrow$ OPN $\uparrow \uparrow$
Foxp3 + ROR $\gamma \mathrm{t} \uparrow$ ROR $\alpha$ 个个 IL-17A/F +/Id $2 \uparrow$ IL-2 $\uparrow$ Foxp3 -

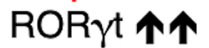
ROR $\alpha \uparrow$

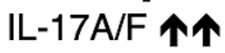
Id2 $\uparrow \uparrow$

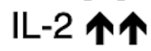

FIGURE 3 | MSC may be responsible for Treg instability and trans-differentiation into Th17 cells. MSC upregulation correlates with the expression of Th17-specific signature molecules, when nTregs are activated in the presence of IL-6 in vitro (Supplementary Figure 1), or in vivo when homeostatic proliferation drives nTregs "parked" in RAG-1-/- mice for 2 weeks to differentiate into two distinct populations, with strong MSC expression marking a metastable Foxp3 $3^{\text {int }}$ population on route to the complete trans-differentiation into a MSC ${ }^{\text {lo }}$ Foxp3 ${ }^{\text {neg }}$ Th17-like population (Supplementary Figure 2), or when FACS-sorted alloantigen-activated iTreg are re-stimulated in mixed lymphocyte reaction (Supplementary Figure 3). MSC and co-expressing Id2 in these cells may interfere with E2A activation of FOXP3 promoter (61). 
CD62 $\mathrm{L}^{\text {lo }} \mathrm{ROR} \gamma \mathrm{t}^{\mathrm{GFP}+}$ cells are either CD4+ or CD4- (52). Compared to CD4+ROR $\gamma \mathrm{t}^{\mathrm{GFP}-}$, both $\mathrm{CD} 4+\mathrm{ROR} \gamma \mathrm{t}^{\mathrm{GFP}+}$ and CD4-ROR t $^{\mathrm{GFP}+}$ cells express high levels of MSC, as well as ROR $\gamma t$, ROR $\alpha$ and Id2 (52). While CD4+ROR $\mathrm{t}^{\mathrm{GFP}+} \mathrm{LTi} / \mathrm{Th} 17$ cells express IL-22 and IL-17A, CD4-ROR $\mathrm{t}^{\mathrm{GFP}+}$ cells express more IL-22, ROR $\gamma$ t and ROR $\alpha$, but no IL-17A (52), suggesting they contain IL-22-secreting ILC3s (a.k.a. NK22 cells or ILC22s). Thus, MSC is uniquely co-expressed with transcription factors ROR $\gamma t / R O R \alpha / I d 2$ in CD4+IL-17A+ (LTi/Th17) cells, as well as in CD4-IL-17A ${ }^{-/ l o}$ populations, the latter of which contains IL22-producing ILC3s (52). Taken together, MSC is expressed not only in freshly induced Th17 cells, with MSC levels correlated to more committed Th17 phenotypes, but also in ROR $\gamma t+$ IL-22producing ILC3s in the Peyer's patches of naïve mice.

Indeed, MSC has a more profound effect on cells from the GALT (such as PP and mesenteric lymph nodes, MLN) than from the spleen and peripheral lymph nodes, which explains why meaningful differences between MSC+/+ and MSC-/- Th subsets are somewhat elusive when naïve cells of spleen or lymph node origin were used for differentiation. For instance, upon Th17 induction by TGF- $\beta 1$ and IL-6, MACS-enriched CD4+ cells from pooled MLN of MSC-/- mice secreted more IL-22 than the wild type counterparts. Under the same condition though, there was no difference between CD4+ T cells from the spleens of MSC-/- and MSC+/+ mice (52). Moreover, the selective production is more dramatic for IL-22 than for IL-17. In line with this, PP cells from MSC-/- mice express much higher levels of IL-22 than MSC+/+ PP cells, and this difference is greatly amplified during colitis (52). All these results suggest that the elusive effect of MSC is on: 1) a special population of IL-22secreting cells in the GALT, most likely ILC3s, and/or 2) synthesis and secretion of IL-22 cytokine per se. More experiments are needed to distinguish whether MSC is a negative gatekeeper for ILC3 developmental pathway, and/or a checkpoint TF controlling IL-22 synthesis.

\section{DISCUSSION: THE YIN AND YANG OF IL-22 IN COLITIS}

\section{Beneficial Effects of IL-22: on Epithelial Cell Regeneration}

In order to maintain a functioning barrier, all the intestinal epithelial cells are replenished within days by continuously proliferating stem cells residing in the crypt region. Given the role of IL-22 in supporting intestinal LGR5+ stem cell mediated epithelial regeneration (73), as well as its stimulation on the synthesis of antimicrobial peptides (AMPs), it is no surprise that the prevailing view is for IL-22 promoting gastrointestinal health (73-80). For instance, IL-22 plays a beneficial role in acute intestinal infections where rapid repair of colonic epithelium is required, such as infection caused by Citrobacter rodentium (74, 81). Likewise, IL-22 facilitates the restitution of epithelium upon inducing acute colonic injury in dextran sulfate sodium (DSS)induced murine colitis (75). In cancer chemotherapy, doselimiting side effects are mainly caused by toxicity in the mucosal surfaces as a result of their high mitotic index. For example, chemotherapy drug methotrexate can induce acute mucositis featured by damage in the epithelium of small intestines, in which IL-22 plays an important restorative role in this self-resolving condition (76). Taken together, the primary insult in these examples is epithelial disruption, where IL-22 drives epithelial cell proliferation and restitution, restoring epithelial barrier. These data have promoted the paradigm of IL-22 being clinically beneficial to repair epithelial damage in human inflammatory bowel disease (IBD), and has led to a clinical trial (NCT02749630), evaluating the role of recombinant IL-22-Fc (Efmarodocokin alfa) in patients with active IBD.

\section{Pathogenic Effects of IL-22: on Immune Dysregulation and ER Stress Response}

However, the key difference between animal models of colitis and human IBD is that, the former usually results from acute insults on the epithelial barrier with self-limiting tissue injury that can quickly resolve upon withdrawal of the insult, whereas the latter is characterized by chronic immune-mediated inflammatory reactions. While IL-22 definitely has a protective role on gut epithelium $(82,83)$, it also promotes colitis in a contextdependent manner $(30,84)$, especially when immune components are involved. For instance, in an adoptive transfer colitis model when Treg-depleted memory/effector CD4+CD45RB ${ }^{\text {lo }} \mathrm{T}$ cells were transferred into RAG-1-/- mice, IL-22 derived from these memory/effector $\mathrm{T}$ cells is pathogenic, whereas IL-22 is protective when CD4+CD45RB hi naïve T cells were transferred (84). Note that in this chronic colitis model, transfer of Treg-depleted CD4+CD45RB ${ }^{\text {lo }}$ T cells into RAG-1-/mice was characterized by a Th $1<$ Th17 cytokine signature in the colon tissues, whereas transfer of CD4+CD45RB hi naïve $\mathrm{T}$ cells demonstrated a Th1 $>$ Th17 cytokine signature (84). In an innate colitis model with anti-CD40 administration in RAG-1-/- mice, IL-22-neutralizing antibody significantly reduced the colon pathology, colitis scores and weight loss triggered by antiCD40 injection, whereas IL-22 plasmid administration exacerbated colitis with infiltration of inflammatory cells and mucosal hyperplasia (30). Interestingly, in that model, the only source of IL-22 in the gut of RAG-1-/- mice is ROR $\gamma \mathrm{t}+$ ILC3s, which are required for colitogenesis (30). Thus, it seems that the pathogenic role of IL-22 could be associated with ILC3s and ROR $\gamma t+$ Th17 cells (e.g., through its soluble factor, IL17A, for chemotaxis of immune cells).

Our own work also supports the notion that heightened IL-22 expression by ILC3s when MSC was deficient has an aggravating effect in DSS-induced murine colitis $(52,85)$. While the wild type mice survived DSS-induced colitis, MSC-/- mice showed increased production of pro-inflammatory cytokines (e.g., IL-22, IL-6, TNF$\alpha$ ) in the gut, with more infiltrating immune cells in colon tissues, reduced body weight, and earlier onset of death. The epithelial injury in the colon tissues was much severer in MSC-/- mice than in the wild type counterpart during colitis, with the counterintuitive observation of higher concentrations of IL-22 in the supernatants of ex vivo cultured colon tissues from the MSC-/group than from the wild type animals $(52,85)$. Surprisingly, 
administration of IL-22BP-Fc, an Fc fusion protein containing the antagonizing IL-22 binding protein (IL-22BP), ameliorated the colitis symptoms in MSC-/- mice (52). This is the first report demonstrating a beneficial effect of IL-22BP in colitis model, where other studies showed the opposite $(86,87)$. Similar to our finding, in an acute polymicrobial sepsis model, IL-22 blockade by IL-22BP-Fc was also shown to be protective against bacteria spread and organ failure (88). Clearly, a balanced production of IL-22BP is context dependent for intestinal homeostasis.

In addition to the animal data, recent human studies also challenge the dogma of IL-22 being beneficial for colitis, especially during chronic inflammation, instead of in acute, self-limiting mucosal injury $(89,90)$. First, IBD is not an acute inflammatory disease. Environmental and genetic factors, as well as gut microbiome, cause stable changes of epithelium functions, which have been considered as the major etiology of IBD (91, 92). Second, a humanized monoclonal antibody (Mab) Risankizumab that specifically blocks IL-23, which triggers IL22 production, demonstrated promising results in a Phase II clinical study in IBD (89). In patients with moderate to severe Crohn's disease (CD) who had failed prior treatment with TNF$\alpha$ blockade, another human IL-23-blocking Mab (MEDI2070) also showed clinical improvement in a Phase 2A study (93). Of note, in that study, patients with higher baseline serum IL-22 levels were more responsive to MEDI2070 (93). More detailed mechanistic studies using colonic epithelial organoids and tissue transcriptome analysis in a large datasets of CD patients revealed that elevated IL-22 orchestrates a pathological endoplasmic reticulum (ER) stress response, amplified by IL-17A (90) which is a cytokine normally co-produced with IL-22 by ILC3s (94).

It should be brought to attention that, in IBD patients, persistently elevated IL-22 levels could alter the baseline of the inflammatory responses in the colonic epithelium. IL-22 induces TNF- $\alpha$, inducible nitric oxide synthetase (iNos2) and apoptosisinducing caspase 12 and STING (90), which can trigger ER stress. ER stress is caused by overwhelmed protein synthesis under adverse stimuli such as infection and non-physiological cellular insults, resulting in the accumulation of aggregated misfolded proteins that are potentially toxic to the cells (95). To mitigate these harmful effects, cells develop a highly conserved protective process called unfolded protein response (UPR). IL-22 significantly stimulates proteins involved in regulating UPR in colonic epithelium (90). For example, IL-22-induced iNos2 is a trigger as well as a downstream effector of the ER stress response (96). In addition, iNos2 causes DNA damage associated colon cancer in chronic colitis (97). IL-22 also significantly upregulates TLR-4 and its signaling adapter MyD88 in colonoids (90). Engagement of this pattern recognition pathway likewise induces ER stress in colonic epithelial stem cells (98). IL-22induced pro-inflammatory molecule STING can increase small intestinal epithelial apoptosis by inducing Type I Interferon production (99). Thus, the role of IL-22 in the context of chronic inflammation during IBD needs to be carefully reevaluated, and the supplementation of exogenous IL-22, especially in an Fc fusion with long serum half-life, to patients with active colitis should be re-considered with extreme caution.

\section{IL-22BP: A Natural Antagonist to Re-Establish Intestinal Homeostasis}

Nature always has checks and balances. A classical example of a naturally-existing cytokine antagonist is interleukin-1 receptor antagonist (IL-1RA). IL-1RA is not an alternatively spliced form of the extracellular domain of any receptor, but is encoded by a separate gene. It binds non-productively to interleukin-1 receptor (IL-1R) and functions as a molecular sink to quench the activities of IL- $1 \alpha$ and IL-1 $\beta$, modulating a variety of IL-1related immune and inflammatory responses (100). In a very similar fashion, IL-22 has its own nemesis: IL-22 binding protein (IL-22BP, a.k.a. IL22RA2) (101, 102). IL-22BP is a soluble receptor, 231 amino acids in length with $34 \%$ sequence homology to IL-22R1 (101, 103), but binds IL-22 with more than 1,000-fold higher affinity than the membrane form IL-22R1 (104) and inhibits IL-22 biological functions by sequestration from its targets (105).

IL-22BP is produced by epithelial cells, eosinophils, macrophages and dendritic cells (106). While IL-22BP expression is significantly increased during IBD, because of the elevated numbers of IL-22BP-producing eosinophils in human gut (86), it should be kept in mind that such increase could be cause or consequence of colonic inflammation, in relation to the surge of IL-22. More relevant to the context of this review, although many reports suggest a detrimental effect of IL-22BP at blocking the protective effects of IL-22 in acute colitis $(75,86$, 87 ), the role of IL-22BP in chronic IBD, in addition to its beneficial effect on blocking IL-22-mediated tumorigenesis (87) and in acute polymicrobial sepsis (88), warrants further investigation. First, species and model differences have produced some contradictory observations. IL-22BP is highly expressed by immature DC in the normal colon but significantly reduced during acute inflammation in DSS-induced murine colitis $(75,107,108)$. In contrast to findings in mice, in patients with IBD increased expression of intestinal IL-22BP was rather observed $(86,87)$. Second, unlike in mice, human IL22BP has three functionally different isoforms, which are generated by alternative splicing (109). Isoform 1 is very poorly secreted and fails to antagonize IL-22 signaling. More recently, isoform 1 was found to be largely retained in ER and functions as an intracellular activator of the UPR response (110). Isoform 2 is the only isoform that is secreted in large amounts when myeloid cells are activated by retinoic acid or Toll-like receptor 2. Isoform 3 is the most widely distributed, but its affinity to IL-22 is 27 -fold lower than that of isoform 2 (109). Thus, human IL-22BP isoforms play distinct temporal and spatial roles fine-tuning the functions of IL-22.

Regardless of its expression profiles and rheostatic regulation on IL-22, IL-22BP is considered a potential therapeutic target. For example, IL-22BP-Fc reduces the severity of cutaneous pathology and inflammation in the mouse model of psoriasis (111). Anti-IL-22BP may have therapeutic value for influenza infection, as a pro-IL-22 environment was shown to alleviate pulmonary inflammation in IL-22BP-knockout mice (IL-22RA2 -/-) during H1N1 (PR8/34 H1N1) infection and protected the lung by promoting tight junction formation (112). It needs to be 
actively pursued whether IL-22BP has therapeutic value for human IBD, in lieu of IL-22-induced colonic epithelial ER stress and completely attenuated chronic colitis in IL-22-/mice or by anti-IL-22 neutralization (90). Testing IL-22BP-Fc as a therapeutic venue in this model and in clinical trials should be a logical next step.

\section{HYPOTHESIS: ROLE OF MSC IN IL-22- MEDIATED PATHOLOGY}

Our own work showed for the first time that exuberant production of IL-22 by GALT cells in the absence of a transcription suppressor, MSC, aggravates colitis even in the acute DSS model $(52,85)$. We also found that during colitis induction by DSS, IL-22RA1 expression in colonic epithelial cells was greatly enhanced in MSC-/- mice compared with $\mathrm{MSC}+/+$ mice, as detected by immunohistochemistry staining (52). Thus, MSC deficiency enforces IL-22 signaling by upregulating both the cytokine and its receptor. Reversal of colitis symptoms by antagonistic IL-22BP-Fc suggested that signaling through supraphysiological amounts of IL-22 and its receptor IL-22RA1 in the gut is detrimental, and could possibly induce colonic epithelial ER stress and consequent apoptosis (90).

Interestingly, we spotted a highly conserved sequence "CCAGCTGC" at +53 nt position downstream of the ATG start codon of both human and mouse IL-22 genes (Figure 4A), which contains an E-box sequence in the center and is only one nt different from the consensus MSC binding motif "CCAGCTGG" (66). Similarly, we also noticed a highly conserved DNA segment containing an E-box sequence "CAGCTG" next to the SP-1 binding site at around -80 nt position upstream of the ATG start codon of both human and mouse IL-22RA1 genes (113) (Figure 4B). Thus, both IL-22 and IL-22RA1 could be yet another set of target genes affected by MSC regulation of E-box activities. A diagram of MSC regulating IL-22 production is proposed (Figure 4C). More studies using gene promoter luciferase reporter assay and EMSA assay are needed to probe whether the potential regulation by MSC on the expression of cytokines and their receptors is a universal phenomenon, or only specific to certain cytokine families, or to cytokines from certain cell subsets.

Besides setting brakes on the expression of cytokine(s) and/ or cytokine receptor(s), it is highly plausible that MSC
A

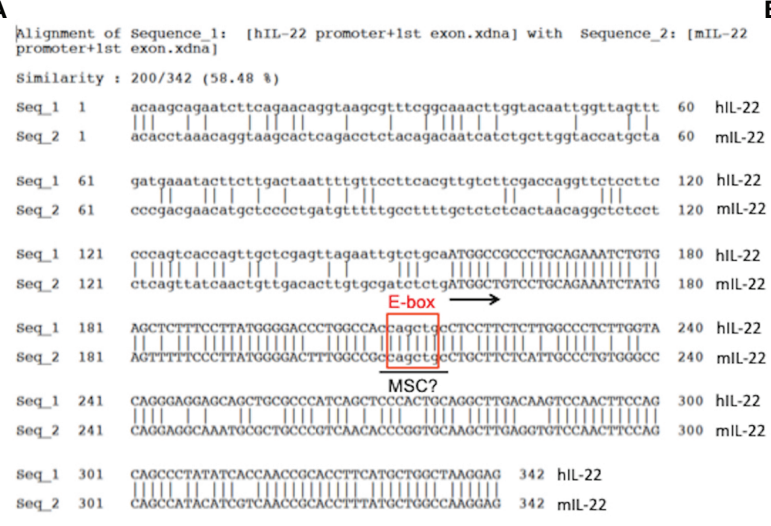

C

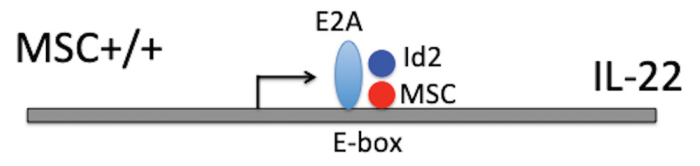

E-box

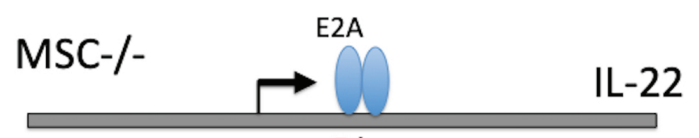

B

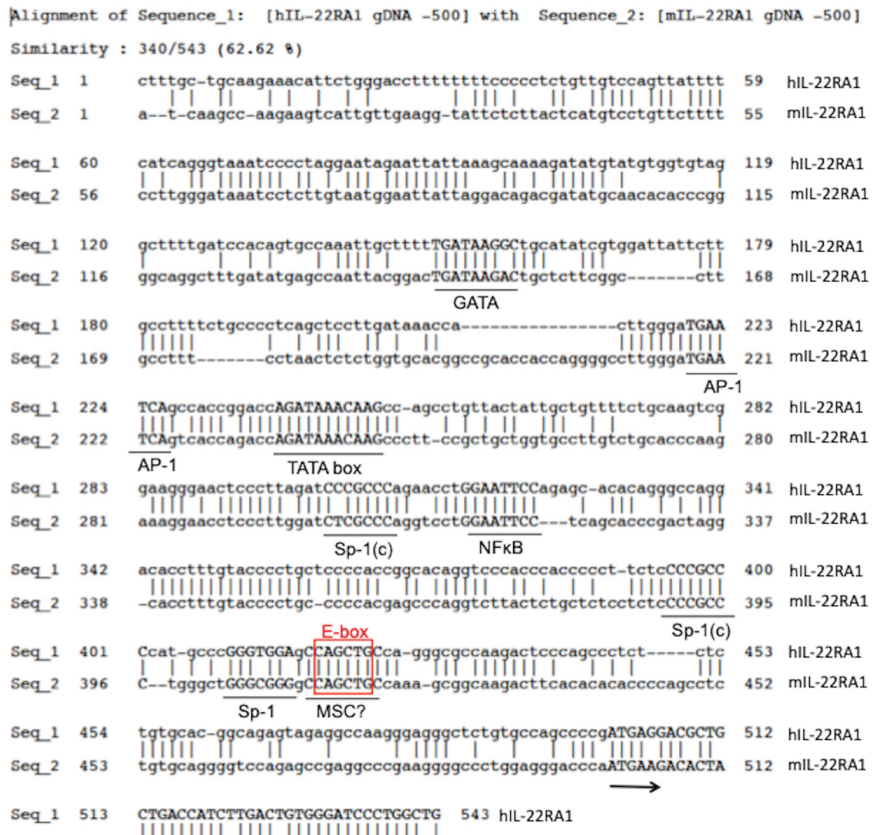

FIGURE 4 | Possible negative regulation of IL-22 and IL-22RA1 by MSC. (A) Alignment of the promoter and the first exon regions of mouse and human IL-22 genes. E2A binding site is boxed, which overlaps with a potential MSC binding motif. Arrow indicates the start of translation. (B) Upstream 500-bp 5' flanking regions of the mouse and human IL-22RA1 genes are aligned to show the putative TATA-boxes and transcription factor binding sites (underlined). Sp-1(c) indicates complementary to a SP-1 binding consensus. E2A binding site is boxed, which overlaps with a potential MSC binding motif. Arrow indicates the start of translation. (C) Diagram of IL-22 transcription regulation by MSC. An E-box motif in the first exon of IL-22 attracts E2A binding and enhancement of IL-22 transcription. This effect is counteracted by MSC : Id2 heterodimer recognizing a similar motif overlapping with the E-box. In the absence of MSC, this negative brake is released, and hence more E2A is bound to the E-box leading to the enhanced transcription and translation of IL-22. A similar scenario may also apply to the transcription regulation of IL-22RA1 by MSC. 
contributes to the differentiation and lineage stability of Th subsets and ILCs. Alternatively, MSC could be involved in stem cell longevity and renewal activity. Regardless of the mechanism, MSC-/- mice provide an unprecedented opportunity to study the potential pathogenic effect of IL-22 in disease models. Through understanding the expression and regulation of MSC per se, we might be able to develop pharmacological agents that act on MSC and/or its expressing cells to re-establish long-lasting intestinal homeostasis (Figure 5).

\section{FUTURE PERSPECTIVES}

The dichotomy of Yin and Yang is the basic concept of Chinese philosophy - viewing the world as not just opposing powers, but

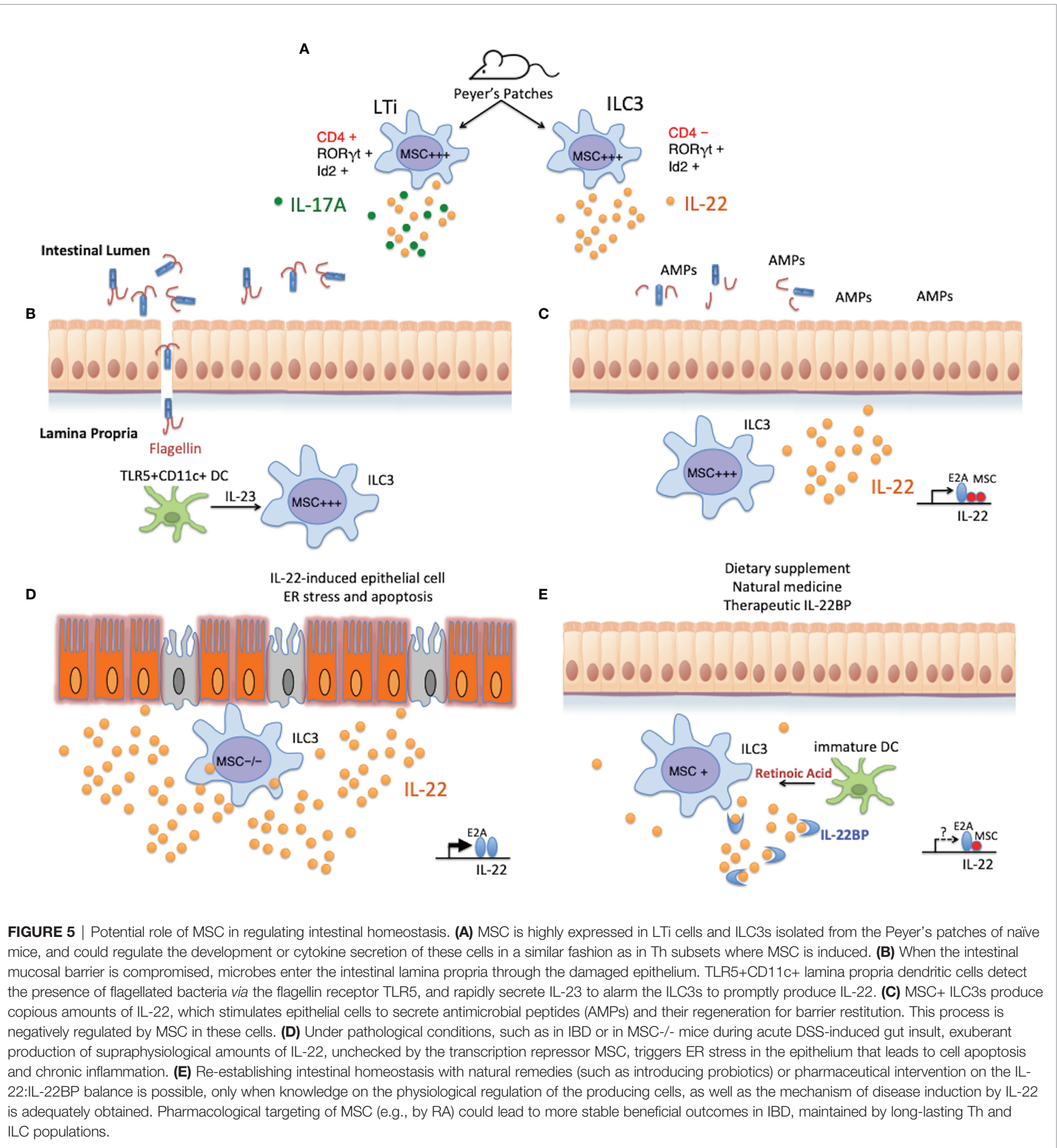


more of dynamic ever-changing elements that can transform into each other under different conditions. IL-22 and IL-22BP are such good examples, i.e., not only IL-22 is a multifaceted cytokine, having diverse beneficial and pathological effects, but also IL-22BP joins the convoluted network of checks and balances. Thus, simple strategy of adding (e.g., IL-22BP-Fc) or removing (e.g., anti-IL-22) elements from the system may be mission-impossible to achieve transformation and ultimate re-establishment of homeostasis. Even worse, such simple strategy when fueled by incomplete understanding of the disease models and mechanisms, such as supplementing IL-22-Fc to IBD patients with chronic inflammation, could have dire consequences.

What we have learned from our studies on transcription factors in general, and MSC in particular, in Th/ILC differentiation and cytokine regulation is that, key regulators more often than not have many downstream targets. Future medicine should consider adopting a strategy to target multiple pathways, perhaps by the assistance of AI and machine learning. For example, the vitamin A metabolite retinoic acid (RA) has been shown to promote Treg but inhibit Th17 differentiation (114). RA also stimulates $\gamma \delta$ T cells and innate lymphoid cells to produce IL-22 and attenuates intestinal inflammation (115). In addition, we found that IL-6-stimulated MSC expression is boosted by IL-23 but suppressed by RA (Supplementary Figure 5). Thus, the attenuating effects of RA on colitis may be linked to its suppression on MSC to release the brake for the expression of physiological levels of IL-22 for cytoprotection. Nevertheless, RA also significantly induces IL-22BP by immature monocyte-derived DC in the gut to antagonize the role of IL-22 (108). A thorough investigation on the multi-effects of RA on wide targets from IL-22, IL-22BP, transcription factors such as MSC, to Treg/Th17/ILC differentiation will gain us a holistic view on how the Yin and Yang forces are working in health and disease, and guide future treatment strategies. Perhaps in another 10 years, AI programs will become powerful enough to assist the analysis from single chemical drugs to multiple modalities as complex as traditional Chinese medicine, which has been tested for thousands of years to be effective in restoration of balance in certain diseases, such as Indigo Naturalis or Qingdai for IBD, where part of the mechanisms seems to involve AHR activation and IL-22 induction $(116,117)$. But then again in colitis or IBD, how much IL-22 is just good, and how much is too much?

\section{REFERENCES}

1. Dumoutier L, Louahed J, Renauld JC. Cloning and Characterization of IL10-Related T Cell-Derived Inducible Factor (IL-TIF), a Novel Cytokine Structurally Related to IL-10 and Inducible by IL-9. J Immunol (2000) 164:1814-9. doi: 10.4049/jimmunol.164.4.1814

2. Keir ME, Yi T, Lu TT, Ghilardi N. The Role of IL-22 in Intestinal Health and Disease. J Exp Med (2020) 217:e20192195. doi: 10.1084/jem.20192195

3. Parks OB, Pociask DA, Hodzic Z, Kolls JK, Good M. Interleukin-22 Signaling in the Regulation of Intestinal Health and Disease. Front Cell Dev Biol (2015) 3:85. doi: 10.3389/fcell.2015.00085

4. Zeng B, Shi S, Ashworth G, Dong C, Liu J, Xing F. ILC3 Function as a Double-Edged Sword in Inflammatory Bowel Diseases. Cell Death Dis (2019) 10:315. doi: 10.1038/s41419-019-1540-2
Can we achieve a rheostatic regulation of IL-22 to restore homeostasis? Such questions can only be answered when the roles of all the molecular and cellular players for IL-22 regulation, including MSC, are sufficiently understood.

\section{DATA AVAILABILITY STATEMENT}

The original contributions presented in the study are included in the article/Supplementary Material. Further inquiries can be directed to the corresponding author.

\section{ETHICS STATEMENT}

The animal study was reviewed and approved by IACUC of Army Medical University.

\section{AUTHOR CONTRIBUTIONS}

JYa, JYu, KL, and YL collected data in own work in colitis studies. $\mathrm{CM}$ contributed with critical reagents used in colitis model, and WG designed research conceptually and wrote the manuscript. All authors have read and agreed to the published version of the manuscript.

\section{FUNDING}

This research was funded by Natural Science Foundation of Chongqing (cstc 2018jcyjAX0258), and Science and Technology Innovation Enhancement Project of Army Medical University (2019XYY22).

\section{SUPPLEMENTARY MATERIAL}

The Supplementary Material for this article can be found online at: https://www.frontiersin.org/articles/10.3389/fimmu.2021. 758730/full\#supplementary-material

5. Shabgah AG, Navashenaq JG, Shabgah OG, Mohammadi H, Sahebkar A. Interleukin-22 in Human Inflammatory Diseases and Viral Infections. Autoimmun Rev (2017) 16:1209-18. doi: 10.1016/j.autrev.2017.10.004

6. Eyerich K, Dimartino V, Cavani A. IL-17 and IL-22 in Immunity: Driving Protection and Pathology. Eur J Immunol (2017) 47:607-14. doi: 10.1002/ eji.201646723

7. Hirahara K, Nakayama T. CD4 + T-Cell Subsets in Inflammatory Diseases: Beyond the Th1/Th2 Paradigm. Int Immunol (2016) 28:163-71. doi: 10.1093/intimm/dxw006

8. Kaplan MH, Hufford MM, Olson MR. The Development and In Vivo Function of T Helper 9 Cells.. Nat Rev Immunol (2015) 15:295-307. doi: 10.1038/nri3824

9. Read KA, Powell MD, Oestreich KJ. T Follicular Helper Cell Programming by Cytokine-Mediated Events. Immunology (2016) 149:253-61. doi: $10.1111 /$ imm. 12648 
10. Eyerich K, Eyerich S. Th22 Cells in Allergic Disease. Allergo J Int (2015) 24:1-7. doi: 10.1007/s40629-015-0039-3

11. Eyerich S, Eyerich K, Pennino D, Carbone T, Nasorri F, Pallotta S, et al. Th22 Cells Represent a Distinct Human T Cell Subset Involved in Epidermal Immunity and Remodeling. J Clin Invest (2009) 119:3573-85. doi: 10.1172/ JCI40202

12. Jia L, Wu C. The Biology and Functions of Th22 Cells. Adv Exp Med Biol (2014) 841:209-30. doi: 10.1007/978-94-017-9487-9_8

13. Azizi G, Yazdani R, Mirshafiey A. Th22 Cells in Autoimmunity: A Review of Current Knowledge. Eur Ann Allergy Clin Immunol (2015) 47:108-17.

14. Bettelli E, Carrier Y, Gao W, Korn T, Strom TB, Oukka M, et al. Reciprocal Developmental Pathways for the Generation of Pathogenic Effector TH17 and Regulatory T Cells. Nature (2006) 441:235-8. doi: 10.1038/nature04753

15. Tait Wojno ED, Artis D. Emerging Concepts and Future Challenges in Innate Lymphoid Cell Biology. J Exp Med (2016) 213:2229-48. doi: 10.1084/ jem. 20160525

16. Bal SM, Golebski K, Spits H. Plasticity of Innate Lymphoid Cell Subsets. Nat Rev Immunol (2020) 20:552-65. doi: 10.1038/s41577-020-0282-9

17. Cherrier M, Sawa S, Eberl G. Notch, Id2, and Roryt Sequentially Orchestrate the Fetal Development of Lymphoid Tissue Inducer Cells. J Exp Med (2012) 209:729-40. doi: 10.1084/jem.20111594

18. Satoh-Takayama N, Lesjean-Pottier S, Vieira P, Sawa S, Eberl G, Vosshenrich CAJ, et al. IL-7 and IL-15 Independently Program the Differentiation of Intestinal CD3-NKp46+ Cell Subsets From Id2-Dependent Precursors. J Exp Med (2010) 207:273-80. doi: 10.1084/jem.20092029

19. Robinette ML, Fuchs A, Cortez VS, Lee JS, Wang Y, Durum SK, et al. Immunological Genome Consortium. Transcriptional Programs Define Molecular Characteristics of Innate Lymphoid Cell Classes and Subsets. Nat Immunol (2015) 16:306-17. doi: 10.1038/ni.3094

20. Vivier E, van de Pavert SA, Cooper MD, Belz GT. The Evolution of Innate Lymphoid Cells. Nat Immunol (2016) 17:790-4. doi: 10.1038/ni.3459

21. Fuchs A, Vermi W, Lee JS, Lonardi S, Gilfillan S, Newberry RD, et al. Intraepithelial Type 1 Innate Lymphoid Cells Are a Unique Subset of IL-12and IL-15-Responsive IFN- $\gamma$-Producing Cells. Immunity (2013) 38:769-81. doi: 10.1016/j.immuni.2013.02.010

22. Mindt BC, Fritz JH, Duerr CU. Group 2 Innate Lymphoid Cells in Pulmonary Immunity and Tissue Homeostasis. Front Immunol (2018) 9:840. doi: 10.3389/fimmu.2018.00840

23. Wang YM, Bakhtiar M, Alexander SI. ILC2: There's a New Cell in Town. JASN (2017) 28:1953-5. doi: 10.1681/ASN.2017040398

24. Takatori H, Kanno Y, Watford WT, Tato CM, Weiss G, Ivanov II, et al. Lymphoid Tissue Inducer-Like Cells Are an Innate Source of IL-17 and IL-22. J Exp Med (2009) 206:35-41. doi: 10.1084/jem.20072713

25. Wang S, Xia P, Chen Y, Qu Y, Xiong Z, Ye B, et al. Regulatory Innate Lymphoid Cells Control Innate Intestinal Inflammation. Cell (2017) 171:201-216.e18. doi: 10.1016/j.cell.2017.07.027

26. Sun H, Wu Y, Zhang Y, Ni B. IL-10-Producing ILCs: Molecular Mechanisms and Disease Relevance. Front Immunol (2021) 12:650200. doi: 10.3389/ fimmu.2021.650200

27. Bando JK, Gilfillan S, Di Luccia B, Fachi JL, Sécca C, Cella M, et al. ILC2s Are the Predominant Source of Intestinal ILC-Derived IL-10. J Exp Med (2020) 217:e20191520. doi: 10.1084/jem.20191520

28. Morita H, Kubo T, Rückert B, Ravindran A, Soyka MB, Rinaldi AO, et al. Induction of Human Regulatory Innate Lymphoid Cells From Group 2 Innate Lymphoid Cells by Retinoic Acid. J Allergy Clin Immunol (2019) 143:2190-201.e9. doi: 10.1016/j.jaci.2018.12.1018

29. Spits H, Di Santo JP. The Expanding Family of Innate Lymphoid Cells: Regulators and Effectors of Immunity and Tissue Remodeling. Nat Immunol (2011) 12:21-7. doi: 10.1038/ni.1962

30. Eken A, Singh AK, Treuting PM, Oukka M. IL-23R+ Innate Lymphoid Cells Induce Colitis via Interleukin-22-Dependent Mechanism. Mucosal Immunol (2014) 7:143-54. doi: 10.1038/mi.2013.33

31. Wang S, Qu Y, Xia P, Chen Y, Zhu X, Zhang J, et al. Transdifferentiation of Tumor Infiltrating Innate Lymphoid Cells During Progression of Colorectal Cancer. Cell Res (2020) 30:610-22. doi: 10.1038/s41422-020-0312-y

32. Ciofani M, Madar A, Galan C, Sellars M, Mace K, Pauli F, et al. A Validated Regulatory Network for Th17 Cell Specification. Cell (2012) 151:289-303. doi: 10.1016/j.cell.2012.09.016
33. Novershtern N, Subramanian A, Lawton LN, Mak RH, Haining WN, McConkey ME, et al. Densely Interconnected Transcriptional Circuits Control Cell States in Human Hematopoiesis. Cell (2011) 144:296-309. doi: 10.1016/j.cell.2011.01.004

34. Korn T, Bettelli E, Gao W, Awasthi A, Jäger A, Strom TB, et al. IL-21 Initiates an Alternative Pathway to Induce Proinflammatory $\mathrm{T}(\mathrm{H}) 17$ Cells. Nature (2007) 448:484-7. doi: 10.1038/nature05970

35. DuPage M, Bluestone JA. Harnessing the Plasticity of CD4(+) T Cells to Treat Immune-Mediated Disease. Nat Rev Immunol (2016) 16:149-63. doi: $10.1038 /$ nri.2015.18

36. Hua J, Inomata $T$, Chen $Y$, Foulsham W, Stevenson W, Shiang T, et al. Pathological Conversion of Regulatory T Cells Is Associated With Loss of Allotolerance. Sci Rep (2018) 8:7059. doi: 10.1038/s41598-018-25384-x

37. Sakaguchi S, Vignali DAA, Rudensky AY, Niec RE, Waldmann H. The Plasticity and Stability of Regulatory T Cells. Nat Rev Immunol (2013) 13:461-7. doi: 10.1038/nri3464

38. Kurschus FC, Croxford AL, Heinen AP, Wörtge S, Ielo D, Waisman A. Genetic Proof for the Transient Nature of the Th17 Phenotype. Eur J Immunol (2010) 40:3336-46. doi: 10.1002/eji.201040755

39. Lee YK, Turner H, Maynard CL, Oliver JR, Chen D, Elson CO, et al. Late Developmental Plasticity in the T Helper 17 Lineage. Immunity (2009) 30:92-107. doi: 10.1016/j.immuni.2008.11.005

40. Annunziato F, Cosmi L, Santarlasci V, Maggi L, Liotta F, Mazzinghi B, et al. Phenotypic and Functional Features of Human Th17 Cells. J Exp Med (2007) 204:1849-61. doi: 10.1084/jem.20070663

41. Downs-Canner S, Berkey S, Delgoffe GM, Edwards RP, Curiel T, Odunsi K, et al. Suppressive IL-17a+Foxp3+ and Ex-Th17 IL-17anegfoxp3+ Treg Cells Are a Source of Tumour-Associated Treg Cells. Nat Commun (2017) 8:14649. doi: 10.1038/ncomms14649

42. Lu J, Webb R, Richardson JA, Olson EN. MyoR: A Muscle-Restricted Basic Helix-Loop-Helix Transcription Factor That Antagonizes the Actions of MyoD. Proc Natl Acad Sci USA (1999) 96:552-7. doi: 10.1073/pnas.96.2.552

43. Robb L, Hartley L, Wang CC, Harvey RP, Begley CG. Musculin: A Murine Basic Helix-Loop-Helix Transcription Factor Gene Expressed in Embryonic Skeletal Muscle. Mech Dev (1998) 76:197-201. doi: 10.1016/S0925-4773(98) 00122-1

44. Wong J, Funes-Duran M, Ahlberg J, Round J, O’Connell R, Miller R, et al. Characterization of a Basic Helix-Loop-Helix Protein, ABF-1: Nuclear Localization, Transcriptional Properties, and Interaction With Id-2. DNA Cell Biol (2001) 20:465-71. doi: 10.1089/104454901316976091

45. Zhao P, Hoffman EP. Musculin Isoforms and Repression of MyoD in Muscle Regeneration. Biochem Biophys Res Commun (2006) 342:835-42. doi: 10.1016/j.bbrc.2006.01.188

46. Hishikawa K, Marumo T, Miura S, Nakanishi A, Matsuzaki Y, Shibata K, et al. Musculin/MyoR Is Expressed in Kidney Side Population Cells and Can Regulate Their Function. J Cell Biol (2005) 169:921-8. doi: 10.1083/jcb.200412167

47. Chiu Y-K, Lin I-Y, Su S-T, Wang K-H, Yang S-Y, Tsai D-Y, et al. Transcription Factor ABF-1 Suppresses Plasma Cell Differentiation But Facilitates Memory B Cell Formation. J Immunol (2014) 193:2207-17. doi: 10.4049/jimmunol.1400411

48. Mathas S, Janz M, Hummel F, Hummel M, Wollert-Wulf B, Lusatis S, et al. Intrinsic Inhibition of Transcription Factor E2A by HLH Proteins ABF-1 and Id2 Mediates Reprogramming of Neoplastic B Cells in Hodgkin Lymphoma. Nat Immunol (2006) 7:207-15. doi: 10.1038/ni1285

49. Debuisson D, Mari N, Denanglaire S, Leo O, Andris F. Myor/ABF-1 mRNA [Corrected] Expression Marks Follicular Helper T Cells But Is Dispensable for Tfh Cell Differentiation and Function In Vivo. PloS One (2013) 8:e84415. doi: 10.1371/journal.pone.0084415

50. Santarlasci V, Mazzoni A, Capone M, Rossi MC, Maggi L, Montaini G, et al. Musculin Inhibits Human T-Helper 17 Cell Response to Interleukin 2 by Controlling STAT5B Activity. Eur J Immunol (2017) 47:1427-42. doi: $10.1002 /$ eji.201746996

51. Wu C, Chen Z, Dardalhon V, Xiao S, Thalhamer T, Liao M, et al. The Transcription Factor Musculin Promotes the Unidirectional Development of Peripheral Treg Cells by Suppressing the TH2 Transcriptional Program. Nat Immunol (2017) 18:344-53. doi: 10.1038/ni.3667

52. Yan J, Yu J, Yuan S, Tang W, Ma W, Yang X, et al. Musculin Is Highly Enriched in Th17 and IL-22-Producing ILC3s and Restrains Pro- 
Inflammatory Cytokines in Murine Colitis. Eur J Immunol (2021) 51:995-8. doi: $10.1002 /$ eji.202048573

53. Zhang F, Fuss IJ, Yang Z, Strober W. Transcription of Roryt in Developing Th17 Cells Is Regulated by E-Proteins. Mucosal Immunol (2014) 7:521-32. doi: $10.1038 / \mathrm{mi} .2013 .69$

54. Kee BL E. And ID Proteins Branch Out. Nat Rev Immunol (2009) 9:175-84. doi: $10.1038 /$ nri2507

55. Verykokakis M, Zook EC, Kee BL. Id'ing Innate and Innate-Like Lymphoid Cells. Immunol Rev (2014) 261:177-97. doi: 10.1111/imr.12203

56. Miyazaki M, Rivera RR, Miyazaki K, Lin YC, Agata Y, Murre C. The Opposing Roles of the Transcription Factor E2A and Its Antagonist Id3 That Orchestrate and Enforce the Naive Fate of T Cells. Nat Immunol (2011) 12:992-1001. doi: 10.1038/ni.2086

57. de Pooter RF, Kee BL. E Proteins and the Regulation of Early Lymphocyte Development. Immunol Rev (2010) 238:93-109. doi: 10.1111/j.1600065X.2010.00957.x

58. Yokota Y, Mansouri A, Mori S, Sugawara S, Adachi S, Nishikawa S, et al. Development of Peripheral Lymphoid Organs and Natural Killer Cells Depends on the Helix-Loop-Helix Inhibitor Id2. Nature (1999) 397:702-6. doi: $10.1038 / 17812$

59. Rivera RR, Johns CP, Quan J, Johnson RS, Murre C. Thymocyte Selection Is Regulated by the Helix-Loop-Helix Inhibitor Protein, Id3. Immunity (2000) 12:17-26. doi: 10.1016/s1074-7613(00)80155-7

60. Maruyama T, Li J, Vaque JP, Konkel JE, Wang W, Zhang B, et al. Control of the Differentiation of Regulatory T Cells and T H 17 Cells by the DNA-Binding Inhibitor Id3. Nat Immunol (2011) 12:86-95. doi: $10.1038 /$ ni.1965

61. Hwang S-M, Sharma G, Verma R, Byun S, Rudra D, Im S-H. InflammationInduced Id2 Promotes Plasticity in Regulatory T Cells. Nat Commun (2018) 9:4736. doi: 10.1038/s41467-018-07254-2

62. Lafita-Navarro MC, Kim M, Borenstein-Auerbach N, Venkateswaran N, Hao Y-H, Ray R, et al. The Aryl Hydrocarbon Receptor Regulates Nucleolar Activity and Protein Synthesis in MYC-Expressing Cells. Genes Dev (2018) 32:1303-8. doi: 10.1101/gad.313007.118

63. Lebel R, McDuff F-O, Lavigne P, Grandbois M. Direct Visualization of the Binding of C-Myc/Max Heterodimeric B-HLH-LZ to E-Box Sequences on the hTERT Promoter. Biochemistry (2007) 46:10279-86. doi: 10.1021/ bi700076m

64. Menning A, Höpken UE, Siegmund K, Lipp M, Hamann A, Huehn J. Distinctive Role of CCR7 in Migration and Functional Activity of Naive- and Effector/Memory-Like Treg Subsets. Eur J Immunol (2007) 37:1575-83. doi: $10.1002 /$ eji.200737201

65. Dias S, Månsson R, Gurbuxani S, Sigvardsson M, Kee BL. E2A Proteins Promote Development of Lymphoid-Primed Multipotent Progenitors. Immunity (2008) 29:217-27. doi: 10.1016/j.immuni.2008.05.015

66. MacQuarrie KL, Yao Z, Fong AP, Tapscott SJ. Genome-Wide Binding of the Basic Helix-Loop-Helix Myogenic Inhibitor Musculin has Substantial Overlap With MyoD: Implications for Buffering Activity. Skelet Muscle (2013) 3:26. doi: 10.1186/2044-5040-3-26

67. Pezzolesi MG, Zbuk KM, Waite KA, Eng C. Comparative Genomic and Functional Analyses Reveal a Novel Cis-Acting PTEN Regulatory Element as a Highly Conserved Functional E-Box Motif Deleted in Cowden Syndrome. Hum Mol Genet (2007) 16:1058-71. doi: 10.1093/hmg/ddm053

68. Dardalhon V, Awasthi A, Kwon H, Galileos G, Gao W, Sobel RA, et al. IL-4 Inhibits TGF-Beta-Induced Foxp3+ T Cells and, Together With TGF-Beta, Generates IL-9+ IL-10+ Foxp3(-) Effector T Cells. Nat Immunol (2008) 9:1347-55. doi: 10.1038/ni.1677

69. Kiss EA, Diefenbach A. Role of the Aryl Hydrocarbon Receptor in Controlling Maintenance and Functional Programs of Roryt+ Innate Lymphoid Cells and Intraepithelial Lymphocytes. Front Immunol (2012) 0:124. doi: $10.3389 /$ fimmu.2012.00124

70. Sawa S, Cherrier M, Lochner M, Satoh-Takayama N, Fehling HJ, Langa F, et al. Lineage Relationship Analysis of RORgammat+ Innate Lymphoid Cells. Science (2010) 330:665-9. doi: 10.1126/science.1194597

71. Xu W, Cherrier DE, Chea S, Vosshenrich C, Serafini N, Petit M, et al. An Id2RFP-Reporter Mouse Redefines Innate Lymphoid Cell Precursor Potentials. Immunity (2019) 50:1054-68.e3. doi: 10.1016/j.immuni. 2019.02.022
72. Eberl G, Marmon S, Sunshine M-J, Rennert PD, Choi Y, Littman DR. An Essential Function for the Nuclear Receptor RORgamma(t) in the Generation of Fetal Lymphoid Tissue Inducer Cells. Nat Immunol (2004) 5:64-73. doi: $10.1038 /$ ni1022

73. Lindemans CA, Calafiore M, Mertelsmann AM, O'Connor MH, Dudakov JA, Jenq RR, et al. Interleukin-22 Promotes Intestinal-Stem-Cell-Mediated Epithelial Regeneration. Nature (2015) 528:560-4. doi: 10.1038/nature16460

74. Satoh-Takayama N, Vosshenrich CAJ, Lesjean-Pottier S, Sawa S, Lochner M, Rattis F, et al. Microbial Flora Drives Interleukin 22 Production in Intestinal NKp46+ Cells That Provide Innate Mucosal Immune Defense. Immunity (2008) 29:958-70. doi: 10.1016/j.immuni.2008.11.001

75. Sugimoto K, Ogawa A, Mizoguchi E, Shimomura Y, Andoh A, Bhan AK, et al. IL-22 Ameliorates Intestinal Inflammation in a Mouse Model of Ulcerative Colitis. J Clin Invest (2008) 118:534-44. doi: 10.1172/JCI33194

76. Aparicio-Domingo P, Romera-Hernandez M, Karrich JJ, Cornelissen F, Papazian N, Lindenbergh-Kortleve DJ, et al. Type 3 Innate Lymphoid Cells Maintain Intestinal Epithelial Stem Cells After Tissue Damage. J Exp Med (2015) 212:1783-91. doi: 10.1084/jem.20150318

77. Zheng Y, Valdez PA, Danilenko DM, Hu Y, Sa SM, Gong Q, et al. Interleukin-22 Mediates Early Host Defense Against Attaching and Effacing Bacterial Pathogens. Nat Med (2008) 14:282-9. doi: 10.1038/ nm1720

78. Sonnenberg GF, Monticelli LA, Elloso MM, Fouser LA, Artis D. CD4(+) Lymphoid Tissue-Inducer Cells Promote Innate Immunity in the Gut. Immunity (2011) 34:122-34. doi: 10.1016/j.immuni.2010.12.009

79. Pickert G, Neufert C, Leppkes M, Zheng Y, Wittkopf N, Warntjen M, et al. STAT3 Links IL-22 Signaling in Intestinal Epithelial Cells to Mucosal Wound Healing. J Exp Med (2009) 206:1465-72. doi: 10.1084/jem.20082683

80. Pham TAN, Clare S, Goulding D, Arasteh JM, Stares MD, Browne HP, et al. Epithelial IL-22RA1-Mediated Fucosylation Promotes Intestinal Colonization Resistance to an Opportunistic Pathogen. Cell Host Microbe (2014) 16:504-16. doi: 10.1016/j.chom.2014.08.017

81. Sanos SL, Bui VL, Mortha A, Oberle K, Heners C, Johner C, et al. RORgammat and Commensal Microflora Are Required for the Differentiation of Mucosal Interleukin 22-Producing NKp46+ Cells. Nat Immunol (2009) 10:83-91. doi: 10.1038/ni.1684

82. Sonnenberg GF, Fouser LA, Artis D. Border Patrol: Regulation of Immunity, Inflammation and Tissue Homeostasis at Barrier Surfaces by IL-22. Nat Immunol (2011) 12:383-90. doi: 10.1038/ni.2025

83. Zenewicz LA, Yancopoulos GD, Valenzuela DM, Murphy AJ, Stevens S, Flavell RA. Innate and Adaptive Interleukin-22 Protects Mice From Inflammatory Bowel Disease. Immunity (2008) 29:947-57. doi: 10.1016/ j.immuni.2008.11.003

84. Kamanaka M, Huber S, Zenewicz LA, Gagliani N, Rathinam C, O'Connor W, et al. Memory/effector (CD45RB(lo)) CD4 T Cells Are Controlled Directly by IL-10 and Cause IL-22-Dependent Intestinal Pathology. J Exp Med (2011) 208:1027-40. doi: 10.1084/jem.20102149

85. Yu J, Liu Y, Zhang W, Yang X, Tang W, Liang H, et al. Musculin Deficiency Aggravates Colonic Injury and Inflammation in Mice With Inflammatory Bowel Disease. Inflammation (2020) 43:1455-63. doi: 10.1007/s10753-02001223-y

86. Martin JC, Bériou G, Heslan M, Bossard C, Jarry A, Abidi A, et al. IL-22BP Is Produced by Eosinophils in Human Gut and Blocks IL-22 Protective Actions During Colitis. Mucosal Immunol (2016) 9:539-49. doi: 10.1038/mi.2015.83

87. Pelczar P, Witkowski M, Perez LG, Kempski J, Hammel AG, Brockmann L, et al. A Pathogenic Role for T Cell-Derived IL-22BP in Inflammatory Bowel Disease. Science (2016) 354:358-62. doi: 10.1126/science.aah5903

88. Weber GF, Schlautkötter S, Kaiser-Moore S, Altmayr F, Holzmann B, Weighardt H. Inhibition of Interleukin-22 Attenuates Bacterial Load and Organ Failure During Acute Polymicrobial Sepsis. Infect Immun (2007) 75:1690-7. doi: 10.1128/IAI.01564-06

89. Feagan BG, Sandborn WJ, D’Haens G, Panés J, Kaser A, Ferrante M, et al. Induction Therapy With the Selective Interleukin-23 Inhibitor Risankizumab in Patients With Moderate-to-Severe Crohn's Disease: A Randomised, Double-Blind, Placebo-Controlled Phase 2 Study. Lancet (2017) 389:1699-709. doi: 10.1016/S0140-6736(17)30570-6

90. Powell N, Pantazi E, Pavlidis P, Tsakmaki A, Li K, Yang F, et al. Interleukin22 Orchestrates a Pathological Endoplasmic Reticulum Stress Response 
Transcriptional Programme in Colonic Epithelial Cells. Gut (2020) 69:57890. doi: 10.1136/gutjnl-2019-318483

91. Ko JK, Auyeung KK. Inflammatory Bowel Disease: Etiology, Pathogenesis and Current Therapy. Curr Pharm Des (2014) 20:1082-96. doi: 10.2174/ 13816128113199990416

92. Kellermayer R, Zilbauer M. The Gut Microbiome and the Triple Environmental Hit Concept of Inflammatory Bowel Disease Pathogenesis. J Pediatr Gastroenterol Nutr (2020) 71:589-95. doi: 10.1097/MPG. 0000000000002908

93. Sands BE, Chen J, Feagan BG, Penney M, Rees WA, Danese S, et al. Efficacy and Safety of MEDI2070, an Antibody Against Interleukin 23, in Patients With Moderate to Severe Crohn's Disease: A Phase 2a Study. Gastroenterology (2017) 153:77-86.e6. doi: 10.1053/j.gastro.2017.03.049

94. Powell N, Lo JW, Biancheri P, Vossenkämper A, Pantazi E, Walker AW, et al. Interleukin 6 Increases Production of Cytokines by Colonic Innate Lymphoid Cells in Mice and Patients With Chronic Intestinal Inflammation. Gastroenterology (2015) 149:456-67.e15. doi: 10.1053/j.gastro.2015.04.017

95. Hiramatsu N, Chiang W-C, Kurt TD, Sigurdson CJ, Lin JH. Multiple Mechanisms of Unfolded Protein Response-Induced Cell Death. Am J Pathol (2015) 185:1800-8. doi: 10.1016/j.ajpath.2015.03.009

96. Yang L, Calay ES, Fan J, Arduini A, Kunz RC, Gygi SP, et al. METABOLISM. S-Nitrosylation Links Obesity-Associated Inflammation to Endoplasmic Reticulum Dysfunction. Science (2015) 349:500-6. doi: 10.1126/science. aaa0079

97. Wang C, Gong G, Sheh A, Muthupalani S, Bryant EM, Puglisi DA, et al. Interleukin-22 Drives Nitric Oxide-Dependent DNA Damage and Dysplasia in a Murine Model of Colitis-Associated Cancer. Mucosal Immunol (2017) 10:1504-17. doi: 10.1038/mi.2017.9

98. Afrazi A, Branca MF, Sodhi CP, Good M, Yamaguchi Y, Egan CE, et al. TollLike Receptor 4-Mediated Endoplasmic Reticulum Stress in Intestinal Crypts Induces Necrotizing Enterocolitis. J Biol Chem (2014) 289:9584-99. doi: $10.1074 /$ jbc.M113.526517

99. Aden K, Tran F, Ito G, Sheibani-Tezerji R, Lipinski S, Kuiper JW, et al. ATG16L1 Orchestrates Interleukin-22 Signaling in the Intestinal Epithelium. Via cGAS-STING J Exp Med (2018) 215:2868-86. doi: 10.1084/jem.20171029

100. Perrier S, Darakhshan F, Hajduch E. IL-1 Receptor Antagonist in Metabolic Diseases: Dr Jekyll or Mr Hyde? FEBS Lett (2006) 580:6289-94. doi: 10.1016/ j.febslet.2006.10.061

101. Dumoutier L, Lejeune D, Colau D, Renauld JC. Cloning and Characterization of IL-22 Binding Protein, a Natural Antagonist of IL-10Related T Cell-Derived Inducible Factor/IL-22. J Immunol (2001) 166:70905. doi: 10.4049/jimmunol.166.12.7090

102. Kotenko SV, Izotova LS, Mirochnitchenko OV, Esterova E, Dickensheets H, Donnelly RP, et al. Identification, Cloning, and Characterization of a Novel Soluble Receptor That Binds IL-22 and Neutralizes its Activity. J Immunol (2001) 166:7096-103. doi: 10.4049/jimmunol.166.12.7096

103. Wei C-C, Ho T-W, Liang W-G, Chen G-Y, Chang M-S. Cloning and Characterization of Mouse IL-22 Binding Protein. Genes Immun (2003) 4:204-11. doi: $10.1038 /$ s..gene.6363947

104. Jones BC, Logsdon NJ, Walter MR. Structure of IL-22 Bound to its HighAffinity IL-22R1 Chain. Structure (2008) 16:1333-44. doi: 10.1016/ j.str.2008.06.005

105. de Moura PR, Watanabe L, Bleicher L, Colau D, Dumoutier L, Lemaire MM, et al. Crystal Structure of a Soluble Decoy Receptor IL-22BP Bound to Interleukin-22. FEBS Lett (2009) 583:1072-7. doi: 10.1016/j.febslet. 2009.03.006

106. Perusina Lanfranca M, Lin Y, Fang J, Zou W, Frankel T. Biological and Pathological Activities of Interleukin-22. J Mol Med (Berl) (2016) 94:523-34. doi: 10.1007/s00109-016-1391-6
107. Huber S, Gagliani N, Zenewicz LA, Huber FJ, Bosurgi L, Hu B, et al. IL-22BP Is Regulated by the Inflammasome and Modulates Tumorigenesis in the Intestine. Nature (2012) 491:259-63. doi: 10.1038/nature11535

108. Martin JCJ, Bériou G, Heslan M, Chauvin C, Utriainen L, Aumeunier A, et al. Interleukin-22 Binding Protein (IL-22BP) Is Constitutively Expressed by a Subset of Conventional Dendritic Cells and Is Strongly Induced by Retinoic Acid. Mucosal Immunol (2014) 7:101-13. doi: 10.1038/mi.2013.28

109. Lim C, Hong M, Savan R. Human IL-22 Binding Protein Isoforms Act as a Rheostat for IL-22 Signaling. Sci Signal (2016) 9:ra95. doi: 10.1126/ scisignal.aad 9887

110. Gómez-Fernández P, Urtasun A, Paton AW, Paton JC, Borrego F, Dersh D, et al. Long Interleukin-22 Binding Protein Isoform-1 Is an Intracellular Activator of the Unfolded Protein Response. Front Immunol (2018) 9:2934. doi: $10.3389 /$ fimmu.2018.02934

111. Fukaya T, Fukui T, Uto T, Takagi H, Nasu J, Miyanaga N, et al. Pivotal Role of IL-22 Binding Protein in the Epithelial Autoregulation of Interleukin-22 Signaling in the Control of Skin Inflammation. Front Immunol (2018) 0:1418. doi: $10.3389 /$ fimmu.2018.01418

112. Hebert KD, Mclaughlin N, Galeas-Pena M, Zhang Z, Eddens T, Govero A, et al. Targeting the IL-22/IL-22BP Axis Enhances Tight Junctions and Reduces Inflammation During Influenza Infection. Mucosal Immunol (2020) 13:64-74. doi: 10.1038/s41385-019-0206-9

113. Tachiiri A, Imamura R, Wang Y, Fukui M, Umemura M, Suda T. Genomic Structure and Inducible Expression of the IL-22 Receptor $\alpha$ Chain in Mice. Genes Immun (2003) 4:153-9. doi: 10.1038/sj.gene.6363934

114. Mucida D, Park Y, Kim G, Turovskaya O, Scott I, Kronenberg M, et al. Reciprocal TH17 and Regulatory T Cell Differentiation Mediated by Retinoic Acid. Science (2007) 317:256-60. doi: 10.1126/science.1145697

115. Mielke LA, Jones SA, Raverdeau M, Higgs R, Stefanska A, Groom JR, et al. Retinoic Acid Expression Associates With Enhanced IL-22 Production by $\gamma \delta$ $\mathrm{T}$ Cells and Innate Lymphoid Cells and Attenuation of Intestinal Inflammation. J Exp Med (2013) 210:1117-24. doi: 10.1084/jem.20121588

116. Naganuma M, Sugimoto S, Mitsuyama K, Kobayashi T, Yoshimura N, Ohi $\mathrm{H}$, et al. Efficacy of Indigo Naturalis in a Multicenter Randomized Controlled Trial of Patients With Ulcerative Colitis. Gastroenterology (2018) 154:93547. doi: 10.1053/j.gastro.2017.11.024

117. Gu S, Xue Y, Gao Y, Shen S, Zhang Y, Chen K, et al. Mechanisms of Indigo Naturalis on Treating Ulcerative Colitis Explored by GEO Gene Chips Combined With Network Pharmacology and Molecular Docking. Sci Rep (2020) 10:15204. doi: 10.1038/s41598-020-71030-w

Conflict of Interest: Authors CM and WG are employed by Antagen Pharmaceuticals, Inc.

The remaining authors declare that the research was conducted in the absence of any commercial or financial relationships that could be construed as a potential conflict of interest.

Publisher's Note: All claims expressed in this article are solely those of the authors and do not necessarily represent those of their affiliated organizations, or those of the publisher, the editors and the reviewers. Any product that may be evaluated in this article, or claim that may be made by its manufacturer, is not guaranteed or endorsed by the publisher.

Copyright (c) 2021 Yan, Yu, Liu, Liu, Mao and Gao. This is an open-access article distributed under the terms of the Creative Commons Attribution License (CC BY). The use, distribution or reproduction in other forums is permitted, provided the original author(s) and the copyright owner(s) are credited and that the original publication in this journal is cited, in accordance with accepted academic practice. No use, distribution or reproduction is permitted which does not comply with these terms. 